\title{
Multiple remissions of extracavitary primary effusion lymphoma treated with a single cycle of liposomal doxorubicin in a patient infected with HIV
}

\author{
J. Chen MD PhD, ${ }^{* \dagger}$ V. Mehraj PhD, ${ }^{* \dagger}$ J. Szabo MD, ${ }^{* \ddagger}$ B. Routy MD PhD, $\$ \|$ R.P. Michel MD CM, ${ }^{*}$ \\ and J.P. Routy $\mathrm{MD}^{*+* *}$
}

\begin{abstract}
Primary effusion lymphoma (PEL) is a rare human herpesvirus 8 (HHV8)-related large B cell lymphoma with plasmablastic, immunoblastic, or anaplastic features that often carries a poor prognosis. This lymphoma occurs mainly in patients with HIV infection, most often with Epstein-Barr virus (EBv) co-infection, and usually presents as body cavity effusions or, less commonly, as extracavitary lesions without effusion (EC-PEL). Chemotherapeutic treatment options are limited and require concurrent antiretroviral therapy (ART).

Here, we report the case of an adult patient with HIv infection and chronic hepatitis E virus (HEv) co-infection who had low CD4 T cell recovery after years of ART. The patient then developed a cutaneous EC-PEL which rapidly regressed after 1 cycle of liposomal doxorubicin (LD) for his Kaposi sarcoma (KS) before treatment with cHOP chemotherapy. He had previously received numerous cycles of LD for cutaneous Ks over 2 years.

Because of the patient's low CD4 T cell count, HEv co-infection, and earlier unexpected remission of EC-PEL before Снор, the patient opted for a single trial of LD before other options. Surprisingly, he experienced a complete remission lasting 18 months. Subsequently, his EC-PEL relapsed twice at 31 and at 41 months after the initial diagnosis. Upon recurrence, a similar single cycle of LD was given, which again induced remission. The patient today is in complete remission after a total of $4 \mathrm{LD}$ infusions over 54 months.

This patient represents a unique case of HIV-with-HHV8-related, EBV-negative EC-PEL with chronic HEV coinfection, in which rapid remission was achieved after a single cycle of LD, suggesting an antiviral response in addition to the chemotherapeutic effect.
\end{abstract}

Key Words HIv, HHV8, primary effusion lymphoma, liposomal doxorubicin, chronic hepatitis E virus infection, Epstein-Barr virus

\section{INTRODUCTION}

Primary effusion lymphoma (PEL), a rare mature B cell non-Hodgkin lymphoma, is a distinct entity that presents as lymphomatous body cavity effusions, generally in the absence of solid tumour masses ${ }^{1,2}$. The diagnosis is based on the presence in the neoplastic cells of human herpesvirus 8 (HHV8), with or without Epstein-Barr virus (EBV), by polymerase chain reaction or immunohistochemistry. Extracavitary PEL (EC-PEL-a solid variant without effusion) has a morphology, immunophenotype, and molecular character similar to those of classical $\mathrm{PEL}^{3}$ and shares PEL's median overall survival of less than 1 year ${ }^{4}$. The disease occurs predominantly in individuals who are immunosuppressed, most often because of HIV infection, and constitutes $5 \%$ of AIDS-related non-Hodgkin lymphomas ${ }^{5}$. Most patients with HIV infection who are receiving antiretroviral therapy (ART) and who develop EC-PEL are treated with CHOP (cyclophosphamide-doxorubicin-vincristine-prednisone) or CHOP-derived regimens ${ }^{4}$.

Here, we report the case of a patient with HIv infection who developed an EBV-negative EC-PEL that was treated with 4 single cycles of liposomal doxorubicin (LD) resulting in 4 complete remissions with a mean relapse time of 13.7 
months, in the context of chronic hepatitis E virus (HEv) infection. To date, our patient remains in complete remission 54 months after the initial diagnosis.

\section{CASE DESCRIPTION}

In February 2012, a 51-year-old white man having sex with men was admitted to the Chronic Viral Illness Service at McGill University Health Centre, Montreal, Quebec, with fever, night sweats, weight loss, and elevated transaminases. He had previously been diagnosed with HIV infection in December 2010 (CD4 T cell count $60 / \mathrm{mm}^{3}$, plasma HIv load $151,000 / \mathrm{mL}$ ) and was being treated with emtricitabine, tenofovir, and raltegravir.

Upon admission in 2012, the patient had bilateral inguinal lymphadenopathy with multiple cutaneous Kaposi sarcoma (Ks) lesions on his thighs, lower legs, and chest, confirmed by biopsies (Table I). After the diagnosis of Ks, the patient was treated with $\mathrm{LD}\left(20 \mathrm{mg} / \mathrm{m}^{2}\right.$ every $2-3$ weeks as determined by response to treatment). The elevation of transaminases was a consequence of HEv infection acquired in Southern France and was treated with oral daily ribavirin $(10 \mathrm{mg} / \mathrm{kg})$, which normalized his transaminases without clearing the infection. The ks lesions waxed and waned, and 31 cycles of LD were given in total. The patient's plasma HIv load remained below the limit of detection, with a modest increase in CD4 T cells.

In May 2014, the patient developed growing grey subcutaneous nodular lesions on his back, neck, and chest, which on biopsy showed diffuse proliferation of large plasmablastic cells (Figure 1). By immunohistochemistry, the cells were positive for HHV8, Bcl2, EMA, MUM1, and lambda light chain, with $100 \%$ Ki-67 staining of lymphoid cellular nuclei. The cells were negative for CD138, CD3, CD10, CD20, CD30, CD45, CD79a, Bcl6, PD-L1, and kappa light chain. In situ hybridization for EBV-related RNA was negative (Figure 2). Those findings were consistent with an EBV-negative EC-PEL.

During this time, the patient experienced new onset of Ks lesions and received 1 cycle of $\mathrm{LD}$, which led to rapid regression of the PEL-related lesions on his back and of the Ks lesions. The patient then received the planned 4 cycles of CHOP for his stage 1E PEL while already in remission after the single cycle of $\mathrm{LD}$.

In November 2015, a left axillary lymph node mass appeared and was biopsied, confirming a recurrence of the EC-PEL. Because of the chronic HEV and the modest CD4 T cell recovery, chemotherapy options were discussed with the patient, including second-line non-Hodgkin lymphoma or LD therapy (based on his unexpected remission before снор). After consideration, the patient opted for a single cycle of LD before the other options. All cutaneous and lymph node lesions disappeared within 2 weeks after that treatment. Complete clinical remission was confirmed 6 months later by a negative positron-emission tomography scan. During his second remission, the patient was treated for his HEV with sofosbuvir (400 mg daily) and ribavirin for 24 weeks, achieving a cure, confirmed by successive negative polymerase chain reaction results.

Thirteen months later, the patient developed a right temporal mass that, on biopsy, was found to be relapsed
EC-PEL. Another complete remission was observed with a cycle of LD. Ten months later, bilateral tonsillar masses appeared, were confirmed to be EC-PEL, and again regressed with a single cycle of LD. To date, the patient remains in complete remission with an increase in his CD4 $\mathrm{T}$ cell count to $150 / \mathrm{mm}^{3}$.

\section{DISCUSSION}

An aggressive mature B cell neoplasm, PEL constitutes approximately $5 \%$ of HIV-related lymphomas ${ }^{1,2,4}$. On microscopy, the malignant cells appear immunoblastic, plasmablastic, or anaplastic ${ }^{3}$. By immunohistochemistry, the cells often reveal prominent features of terminally differentiated plasma cells. Most express CD45, but lack other B cell markers; aberrant T cell-associated antigens are rarely expressed ${ }^{1,2}$. The presence of HHv8 (which is also the causative agent for Ks, HHV8-associated diffuse large B-cell lymphoma, and multicentric Castleman disease ${ }^{6-9}$ ) in the cells is essential to confirm the diagnosis of PEL. Co-infection with EBV is observed in more than $80 \%$ of cases $^{10}$. Primary effusion lymphoma that is EBV-negative is most frequently reported in elderly non-immunocompromised individuals living in the Mediterranean region ${ }^{11}$. Our patient, although EBV-negative by in situ hybridization, showed EBV positivity in blood by polymerase chain reaction.

Given the rarity of EC-PEL, guidelines for optimal treatment are limited. Therapy based on снор chemotherapy is associated with a $40 \%-50 \%$ response rate ${ }^{4}$. The modest improvement in survival for patients receiving ART compared with untreated individuals was attributed to virus control and immune recovery ${ }^{12}$. Historically, median overall survival in EC-PEL is less than 1 year. Surprisingly, our patient achieved several remissions totalling 54 months after the initial diagnosis of EC-PEL. The patient rapidly responded to a single cycle of infusion $\mathrm{LD}$, with remissions ranging from 10 months to 18 months. During the first cycle of LD, when the patient received CHOP, the duration of the remission was not different from the durations achieved after cycles of LD without CHOP. To our knowledge, this patient is the first with EC-PEL to have achieved remission after a single infusion of LD. Two HIV-negative elderly patients with PEL receiving LD with bortezomib and rituximab have been reported, with variable outcomes ${ }^{13,14}$. Treatment with LD was proposed to our patient because of his chronic HEV infection and because treatment for relapsed non-Hodgkin lymphoma with chemotherapy can lead to HEv reactivation and possibly fulminant hepatitis. In a patient with a CD4 T cell count less than $100 / \mathrm{mm}^{3}$, chemotherapy would also worsen immunosuppression ${ }^{15}$.

Hepatitis E is an RNA virus, endemic in certain countries, including Southern France ${ }^{16}$. Hepatitis resulting from HEV is usually self-limiting, but can evolve into a chronic infection in immunosuppressed patients and could be fatal in some cases of lymphoma ${ }^{17}$. In our patient, HEV might have triggered the development of PEL; chronic viral hepatitis has been reported to be associated with lymphoproliferative disorders ${ }^{18,19}$. However, because the cure of HEV in our patient did not prevent recurrence of EC-PEL, we do not consider that HEv contributed directly to the development of the lymphoma. 


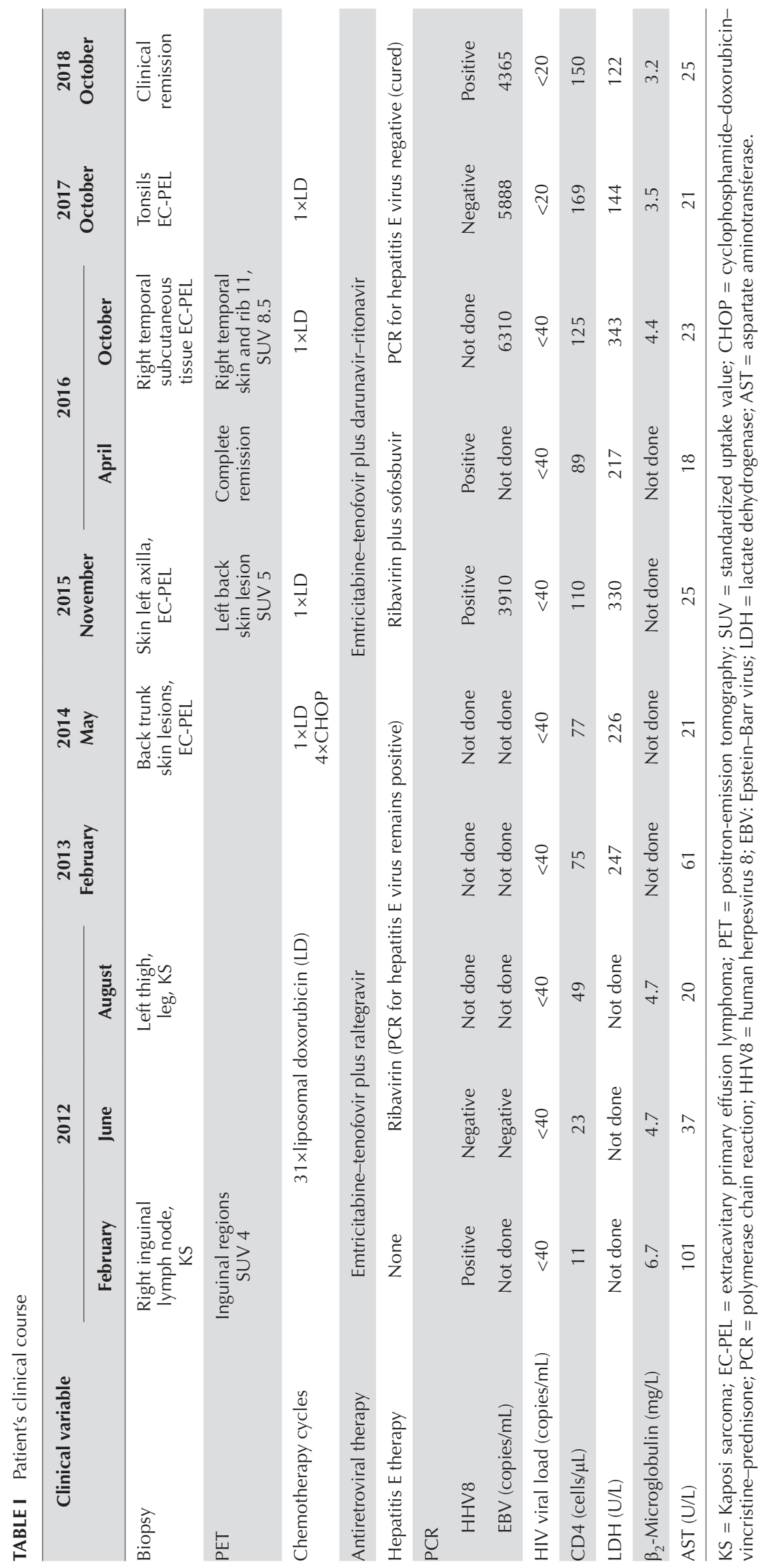




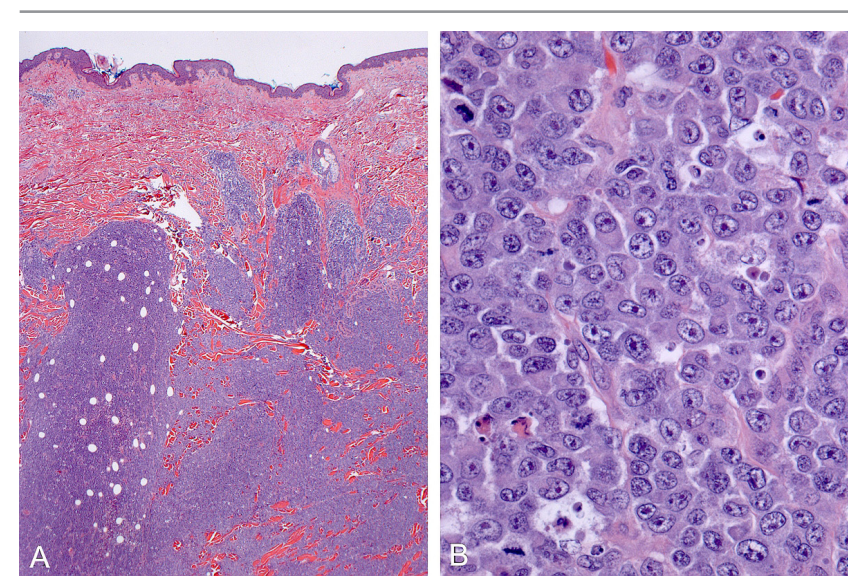

FIGURE 1 Skin biopsy performed in 2014 shows (A) a prominent mid-dermal and deep dermal infiltrate of blue cells, that (B) at high power have a distinctly plasmablastic appearance, characteristic of primary effusion lymphoma.

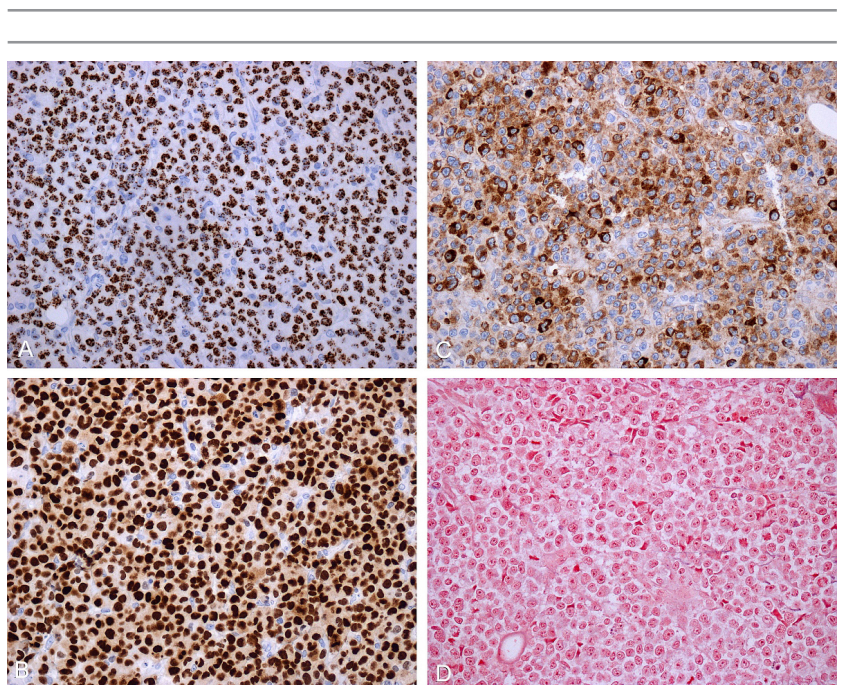

FIGURE 2 Representative immunohistochemical stains show (A) prominent granular nuclear positivity of the extracavitary primary effusion lymphoma for HHV8 (antibody to LANA1), (B) nuclear positivity for MUM1, and (C) cytoplasmic positivity for lambda light chain. (D) In situ hybridization for Epstein-Barr virus (EBER) was negative.

The remission associated with LD might be explained by an increase in the doxorubicin concentration caused by the pegylated liposomal formulation in the target organ, enriching the lymphatic system and preventing it from being engulfed by macrophages and monocytes, thus prolonging the drug's half-life ${ }^{20}$. Another consideration might be that the liposome itself could have interacted with the lipid structure of the envelope of the contributing viruses ${ }^{21,22}$. Because only a single cycle of LD was given, we propose that the chemotherapy might work by interrupting the interaction between HIV and HHV8 rather than by inducing its cytotoxic effects, an action that would therefore not be associated with development of resistance to chemotherapy.

In addition to ART and chemotherapy, herpes antiviral therapy remains an unconfirmed option for treating $\mathrm{PEL}^{23}$. Ribavirin and sofosbuvir were used simultaneously to treat the HEV infection in our patient. Interestingly, ribavirin has shown potential anti-lymphoma activity in vivo ${ }^{24}$. Nevertheless, recurrence has been seen both with and without ribavirin. In addition, $50 \%$ of patients with PEL have been shown to be PD-L1 positive, which makes the use of anti-PD-1 and anti-PD-L1 antibodies a promising therapeutic option in patients with HIV infection ${ }^{25}$. That immune checkpoint blocking approach might control HIV progression without causing further immunosuppression $^{25}$. Immunotherapy was not an option in our case, however, because our patient's biopsy was negative for PD-L1.

To date, our patient remains in complete remission after having received a total of 4 cycles of infusional LD over 54 months, making this case unique, given that median survival for patients with EC-PEL is less than 12 months.

\section{SUMMARY}

We report a case of a patient with HIV infection and chronic HEV infection who developed HHV8-related EC-PEL and who achieved significant remissions after low-toxicity, low-immunosuppressive single cycles of LD, suggesting an antiviral response in addition to a chemotherapeutic effect.

\section{ACKNOWLEDGMENTS}

We thank the patient for his consent and help in reconstituting his complex medical history. We thank Angie Massicotte and Kathleen Nasr for coordination and critical reading of this case report. We are supported by Fonds de recherche Quebec-Santé [FRQ-s (Le réseau SIDA/maladies infectieuses du Québec and ThéCell)]; the Canadian Institutes of Health Research [CIHR (grants MOP 103230 and 154051)]; the Vaccines and Immunotherapies Core of the cIHR Canadian HIV Trials Network (grant CTN 257); the Canadian Foundation for AIDS Research [CANFAR (grant 02-512)]; and the Canadian HIV Cure Enterprise Team Grant (HIG-133050) awarded by CIHR in partnership with CANFAR. JC was supported by a CTN postdoctoral fellowship award. VM was supported by an FRQ-S Postdoctoral Fellowship Award. JPR is the holder of the Louis Lowenstein Chair in Hematology and Oncology, McGill University.

\section{CONFLICT OF INTEREST DISCLOSURES}

We have read and understood Current Oncology's policy on disclosing conflicts of interest, and we declare that we have none.

\section{AUTHOR AFFILIATIONS}

*Chronic Viral Illness Service, McGill University Health Centre, ${ }^{\dagger}$ Research Institute of the McGill University Health Centre, ${ }^{\ddagger}$ Clinique médicale l'Actuel, ${ }^{\S}$ Division of Hemato-oncology, Centre hospitalier de l'Université de Montréal, "Centre de recherche du Centre hospitalier de l'Université de Montréal, "Department of Pathology, McGill University, and **Division of Hematology, McGill University Health Centre, Montreal, QC.

\section{REFERENCES}

1. Said JCE. Primary effusion lymphoma. In: Swerdlow SH, Campo E, Harris NL, et al., eds. WHO Classification of Tumours of Haematopoietic and Lymphoid Tissues. 4th ed. Lyon, France: IARC Publications; 2017: 323-4.

2. Swerdlow SH, Campo E, Pileri SA, et al. The 2016 revision of the World Health Organization classification of lymphoid neoplasms. Blood 2016;127:2375-90.

3. Chadburn A, Hyjek E, Mathew S, Cesarman E, Said J, Knowles DM. KsHv-positive solid lymphomas represent an extracavitary variant of primary effusion lymphoma. Am J Surg Pathol 2004;28:1401-16. 
4. Guillet S, Gerard L, Meignin V, et al. Classic and extracavitary primary effusion lymphoma in $51 \mathrm{HIV}$-infected patients from a single institution. Am J Hematol 2016;91:233-7.

5. Gloghini A, Dolcetti R, Carbone A. Lymphomas occurring specifically in HIV-infected patients: from pathogenesis to pathology. Semin Cancer Biol 2013;23:457-67.

6. Bestawros A, Boulassel MR, Michel RP, Routy JP. HнV-8 linked to Kaposi's sarcoma, Castleman's disease and primary effusion lymphoma in a HIV-1-infected man. J Clin Virol 2008;42:179-81.

7. Bestawros A, Michel R, Seguin C, Routy JP. Multicentric Castleman's disease treated with combination chemotherapy and rituximab in four HIV-positive men: a case series. $A m \mathrm{~J}$ Hematol 2008;83:508-11.

8. Boivin G, Gaudreau A, Routy JP. Evaluation of the human herpesvirus 8 DNA load in blood and Kaposi's sarcoma skin lesions from AIDS patients on highly active antiretroviral therapy. AIDS 2000;14:1907-10.

9. Goncalves PH, Uldrick TS, Yarchoan R. HIV-associated Kaposi sarcoma and related diseases. AIDS 2017;31:1903-16.

10. Yarchoan R, Uldrick TS. HIV-associated cancers and related diseases. NEngl J Med 2018;378:1029-41.

11. Song JY, Jaffe ES. HHV-8-positive but EBV-negative primary effusion lymphoma. Blood 2013;122:3712.

12. Boulanger E, Gerard L, Gabarre J, et al. Prognostic factors and outcome of human herpesvirus 8-associated primary effusion lymphoma in patients with AIDs. J Clin Oncol 2005;23:4372-80.

13. Siddiqi T, Joyce RM. A case of HIV-negative primary effusion lymphoma treated with bortezomib, pegylated liposomal doxorubicin, and rituximab. Clin Lymphoma Myeloma 2008;8:300-4.

14. Christenson ES, Teply B, Agrawal V, Illei P, Gurakar A, Kanakry JA. Human herpesvirus 8-related primary effusion lymphoma after liver transplantation. Am J Transplant 2015;15:2762-6.

15. Rudek MA, Flexner C, Ambinder RF. Use of antineoplastic agents in patients with cancer who have HIV/AIDs. Lancet Oncol 2011;12:905-12.

16. Abravanel $\mathrm{F}$, Lhomme S, Fougere $\mathrm{M}$, et al. Hev infection in French HIV-infected patients. J Infect 2017;74:310-13.

17. Nimgaonkar I, Ding Q, Schwartz RE, Ploss A. Hepatitis E virus: advances and challenges. Nat Rev Gastroenterol Hepatol 2018;15:96-110.

18. Mallet V, Bruneau J, Zuber J, et al. Hepatitis E virus-induced primary cutaneous $\mathrm{CD} 30^{+} \mathrm{T}$ cell lymphoproliferative disorder. J Hepatol 2017;67:1334-9.

19. Pischke S, Hartl J, Pas SD, Lohse AW, Jacobs BC, Van der Eijk AA. Hepatitis E virus: infection beyond the liver? J Hepatol 2017;66:1082-95.

20. Wang Y, Huang W, Hu L, et al. Multicenter study of combination DEP regimen as a salvage therapy for adult refractory hemophagocytic lymphohistiocytosis. Blood 2015;126:2186-92.

21. Tam VC.Lipidomic profiling of bioactivelipids by mass spectrometry during microbial infections. Semin Immunol 2013;25:240-8.

22. Henry BD, Neill DR, Becker KA, et al. Engineered liposomes sequester bacterial exotoxins and protect from severe invasive infections in mice. Nat Biotechnol 2015;33:81-8.

23. Marquet J, Velazquez-Kennedy K, Lopez S, Benito A, Blanchard MJ, Garcia-Vela JA. Case report of a primary effusion lymphoma successfully treated with oral valganciclovir after failing chemotherapy. Hematol Oncol 2018;36:316-19.

24. Rutherford SC, Stewart EN, Chen Z, et al. The eIF4E inhibitor ribavirin as a potential antilymphoma therapeutic: early clinical data. Leuk Lymphoma 2018;59:256-8.

25. Goodman A, Patel SP, Kurzrock R. PD-1-PD-L1 immunecheckpoint blockade in B-cell lymphomas. Nat Rev Clin Oncol 2017;14:203-20. 